\title{
Systems biology analysis reveals role of MDM2 in diabetic nephropathy
}

Rintaro Saito, ${ }^{1,2,3}$ Anaïs Rocanin-Arjo, ${ }^{4}$ Young-Hyun You, ${ }^{2,3}$ Manjula Darshi, ${ }^{1,3}$ Benjamin Van Espen, ${ }^{1,3}$ Satoshi Miyamoto, ${ }^{2,3}$ Jessica Pham, ${ }^{2,3}$ Minya Pu, ${ }^{1,5}$ Simone Romoli, ${ }^{4}$ Loki Natarajan, ${ }^{1,5}$ Wenjun Ju, ${ }^{6}$ Matthias Kretzler, ${ }^{6}$ Robert Nelson, ${ }^{7}$ Keiichiro Ono, ${ }^{3}$ Dana Thomasova, ${ }^{4}$ Shrikant R. Mulay, ${ }^{4}$ Trey Ideker, ${ }^{3}$ Vivette D'Agati, ${ }^{8}$ Ergin Beyret, ${ }^{9}$ Juan Carlos Izpisua Belmonte, ${ }^{9}$ Hans Joachim Anders, ${ }^{4}$ and Kumar Sharma $a^{1,2,3,10}$

IInstitute of Metabolomic Medicine, ${ }^{2}$ Center for Renal Translational Medicine, Division of Nephrology-Hypertension, ${ }^{3}$ Division of Medical Genetics, Department of Medicine, UCSD, San Diego, California, USA. ${ }^{4}$ Medizinische Klinik und Poliklinik IV, Klinikum der Universität München, LMU Munich, Munich, Germany. ${ }^{5}$ Department of Family Medicine and Epidemiology, UCSD, San Diego, California, USA. ${ }^{6}$ Department of Internal Medicine, Nephrology and Department of Computational Medicine and Bioinformatics, University of Michigan, Ann Arbor, Michigan, USA. ${ }^{7}$ National Institute of Diabetes and Digestive and Kidney Diseases, Phoenix, Arizona, USA. ${ }^{8}$ Renal Pathology Laboratory, Columbia University, College of Physicians and Surgeons, Department of Pathology, New York, New York, USA. '9Salk Institute for Biological Studies, San Diego, California, USA. ${ }^{10}$ Veterans Affairs Health Systems, San Diego, California, USA.

To derive new insights in diabetic complications, we integrated publicly available human proteinprotein interaction (PPI) networks with global metabolic networks using metabolomic data from patients with diabetic nephropathy. We focused on the participating proteins in the network that were computationally predicted to connect the urine metabolites. MDM2 had the highest significant number of PPI connections. As validation, significant downregulation of MDM2 gene expression was found in both glomerular and tubulointerstitial compartments of kidney biopsy tissue from 2 independent cohorts of patients with diabetic nephropathy. In diabetic mice, chemical inhibition of MDM2 with Nutlin-3a led to reduction in the number of podocytes, increased blood urea nitrogen, and increased mortality. Addition of Nutlin-3a decreased $W_{T 1^{+}}$cells in embryonic kidneys. Both podocyte- and tubule-specific MDM2-knockout mice exhibited severe glomerular and tubular dysfunction, respectively. Interestingly, the only 2 metabolites that were reduced in both podocyte and tubule-specific MDM2-knockout mice were 3-methylcrotonylglycine and uracil, both of which were also reduced in human diabetic kidney disease. Thus, our bioinformatics tool combined with multi-omics studies identified an important functional role for MDM2 in glomeruli and tubules of the diabetic nephropathic kidney and links MDM2 to a reduction in 2 key metabolite biomarkers.

Conflict of interest: $K$. Sharma is the founder of ClinMet, Inc., was on the Board of Directors, a scientific adviser and held an equity interest. K. Sharma's spouse is a co-founder and was the President and Chief Operating Officer and held an equity interest.

Submitted: April 5, 2016 Accepted: September 21, 2016 Published: October 20, 2016

Reference information: JCI Insight. 2016;1(17):e87877. doi:10.1172/jci.insight.87877.

\section{Introduction}

Metabolomics has become a leading technology to observe cellular states in a comprehensive and potentially high-throughput manner. Other omic technologies such as microarray, next-generation sequencing, and proteomics provide comprehensive information about gene expression and protein levels. However, posttranscriptional and posttranslational modifications of genes and proteins, respectively, do not indicate the direction of change of their underlying function in physiologic and pathophysiologic states. On the contrary, metabolites are the final product of cellular processes, providing a direct link to phenotypes (1). For this reason, an increasing number of studies have been conducted to discover metabolites whose levels significantly change in a specific clinical condition (2-4). In a previous study, we identified a panel of 13 metabolites that were robustly altered in patients with diabetic nephropathy (3), thus motivating us to investigate the underlying molecular networks linked to these metabolites.

By integrating significantly altered metabolites with metabolic pathways using publicly available resources such as KEGG (5), Reactome (6), or MetScape (7), one may be able to identify perturbed parts of biochemical pathways. However, if the significant metabolites are dispersed over multiple pathways, biological interpretation becomes a difficult task. To find possible dominant pathways that are likely to be 
involved in significant changes in individual metabolite levels, metabolite set enrichment analysis (MSEA) and related techniques have been developed $(8,9)$. These approaches may make connections among sets of metabolites that are on the same classical pathways, but often do not identify new connections between metabolites that participate in different pathways. In addition, the fraction of currently known metabolic pathways among all actual metabolic pathways in the cell is limited (10), thus reducing the identification of possible connections between metabolites.

Recent advances in technologies have supported the development of genome-wide protein-protein interaction (PPI) networks (11), which may provide novel connections among enzymes and metabolic pathways. We therefore hypothesized that some proteins linking enzymes associated with reactions involving significant metabolites may act as bridges, constituting less-well-defined pathways. These bridges may then explain connections among metabolites that display quantitative changes in clinical samples. In this work, we present MetBridge, an integrated map of metabolic networks and PPI networks relevant to human biology. By focusing on the 13 metabolites linked to human diabetic kidney disease as an input, our developed software MetBridge Generator extracted a subnetwork $\left(\right.$ MetBridge $_{\mathrm{DKD}}$ ) from MetBridge that potentially regulates these metabolites. We further assessed the potential relevance of key bridge proteins in MetBridge $_{\mathrm{DKD}}$ to diabetic nephropathy by examining gene expression of highly significant bridge proteins. We found that of the top 5 identified proteins that had the greatest number of interactions with the enzymes regulating the 13-metabolite signature of diabetic kidney disease, 4 had significant dysregulation of their gene expression in independent kidney biopsies of patients with diabetic nephropathy. In particular, MDM2 had the greatest number of interacting proteins in the network and had the strongest significant downregulation of gene expression in both glomerular and tubulointerstitial compartments across 2 independent patient cohorts. Functional significance was demonstrated with chemical inhibition of MDM2 and gene knockout in podocytes and tubular epithelial cells in mouse models. Interestingly, 3-methylcrotonylglycine and uracil, which were part of our panel of 13 metabolites, were reduced in the MDM2-knockout mice in both podocytes and tubules, thus indicating partial recapitulation of the metabolite downregulation pattern in patients with diabetic kidney disease.

\section{Results}

Construction of MetBridge network. The global human metabolic map was obtained from the KEGG database (5) and internal database (3) and integrated with human PPI data from the BioGRID database (12). The integrated network initially contained 161,024 edges connecting 16,525 nodes. We restricted nodes to metabolites, their associated enzymes, and proteins that directly interact with these enzymes. The resulting network, designated as "MetBridge", contained 72,343 edges connecting 4,941 nodes. MetBridge was loaded onto Cytoscape (13).

This Cytoscape map provides users a global view of not only metabolic pathways but also possible connections between enzymes through PPIs (Supplemental Figure 1; supplemental material available online with this article; doi:10.1172/jci.insight.87877DS1). There are 3 types of nodes on the network: metabolites, their associated enzymes, and proteins that interact with the enzymes. These proteins are designated as "bridge proteins" - the proteins that connect the enzymes. They are discriminated by the shape and color of the nodes. Edges represent association between metabolites and enzymes, or PPIs, which can be discriminated by their colors. We then developed a Cytoscape App (plugin), designated as "MetBridge Generator", to search for subnetworks that connect a given set of significant metabolites (Supplemental Figure 1). A user can input metabolites of interest by HMDB IDs. The App searches for interacting protein partners of enzymes associated with reactions of given metabolites through the PPI network. The subnetwork containing the metabolites, their associated enzymes, and bridge proteins are extracted and displayed on Cytoscape. The App also assesses the significance value of the number of interactions of bridge proteins to the enzymes regulating the input metabolites compared with the other enzymes on the network (see supplemental methods). The sizes of bridge proteins are proportional to the calculated significance (Figure 1); thus, a user can intuitively understand which bridge proteins have more potential regulatory effects on the given metabolites.

Enriched functions of enzymes and bridge proteins in the subnetwork MetBridge ${ }_{D K D}$. We tested the App using a panel of 13 key metabolites that distinguished patients with diabetic kidney disease (3). With the MetBridge $_{\mathrm{DKD}}$ network, 39 enzymes interacted with the 13 metabolites and 291 bridge proteins in turn interacted with these enzymes (Figure 1). Additionally, 21 enzymes interacted with at least 1 of these 39 enzymes and 


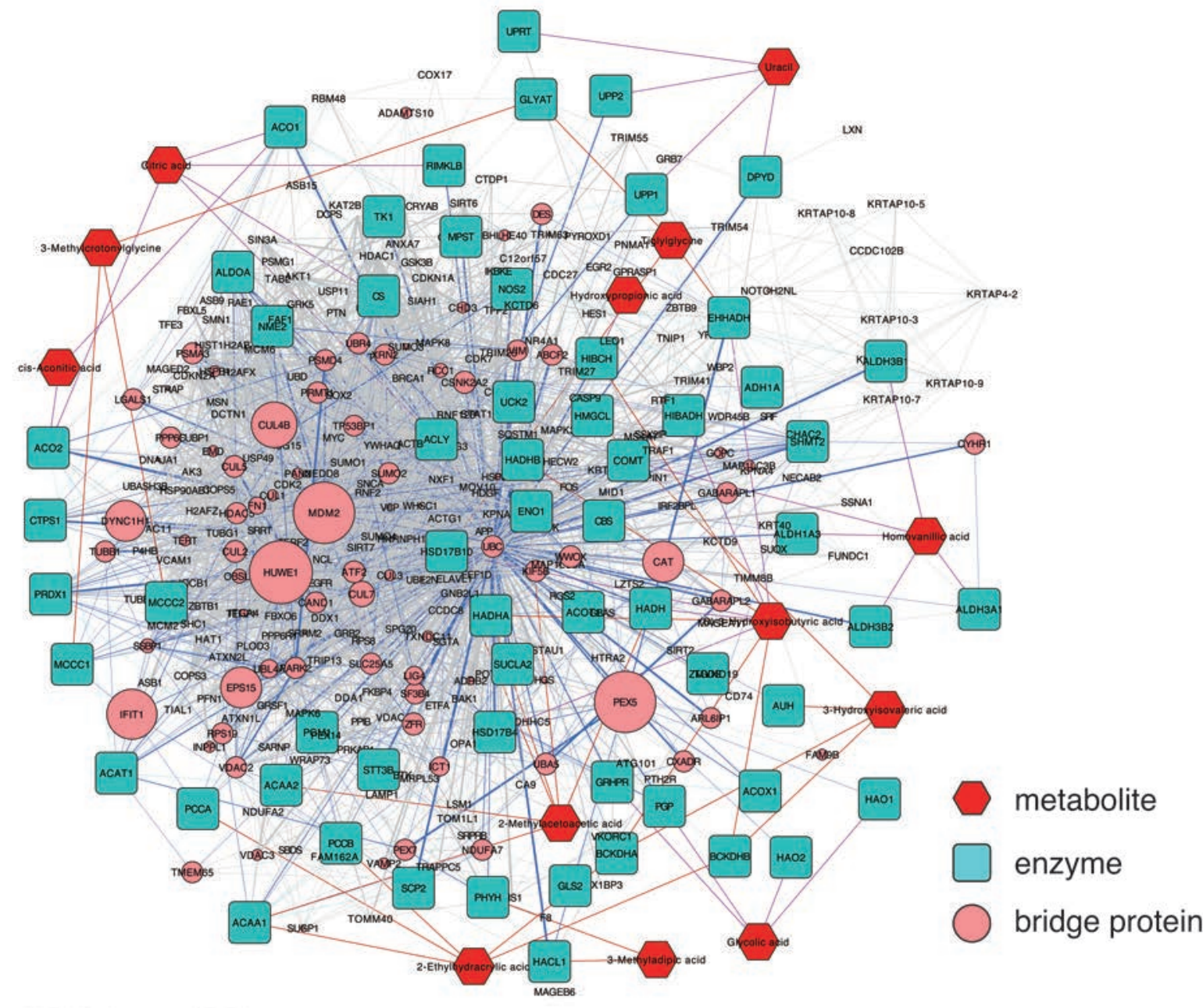

BioGRID-based PPI

between significant bridge proteins

between enzymes

other

\section{Association of metabolite with enzyme KEGG-based \\ Internally curated}

Figure 1. Metabolite protein-protein interaction network (MetBridge ${ }_{\text {око }}$ ) connecting 13 metabolites that were significantly decreased in diabetic kidney disease samples. Red, blue, and pink nodes represent metabolites, enzymes, and bridge proteins, respectively. Pink, red, and blue edges represent KEGG-based associations of metabolite with enzyme, internally curated associations of metabolite with enzyme, and BioGRID-based protein-protein interaction (PPIs), respectively. The network was created using our internally developed MetBridge Generator, which runs as an App on Cytoscape. Node sizes of the bridge proteins (pink) are proportional to significance of node degree. Edge widths of PPIs are proportional to number of articles reporting the corresponding interactions.

at least 1 of these 291 proteins. There were 3,390 edges connecting these nodes, which include 2,591 edges connecting these proteins. If we randomly pick the same number of proteins (291 proteins) from our MetBridge, which contains 58,703 edges among 3,387 proteins, we expect to observe only $\sim 432$ edges among these proteins by chance, which is significantly fewer than the actual number of 2,591 edges $(P<2.33 \times$ $10^{-1,123}$, hypergeometric test; see supplemental methods). Thus, the 13 metabolites were all connected by a modestly extensive PPI network.

We checked the gene expression of these bridge proteins that participate in our MetBridge ${ }_{\mathrm{DKD}}$ network in mouse podocytes. RNAseq data using mouse podocytes were obtained from a previous study (14). Approximately $81.6 \%$ of the transcripts corresponding to these bridge proteins participating in MetBridge ${ }_{\mathrm{DKD}}$ were detected in the podocyte database (based on the threshold of FPKM $>3$ (https://hpcwebapps.cit.nih.gov/ ESBL/Database/Podocyte_Transcriptome/index.htm). The detected transcripts included those corre- 

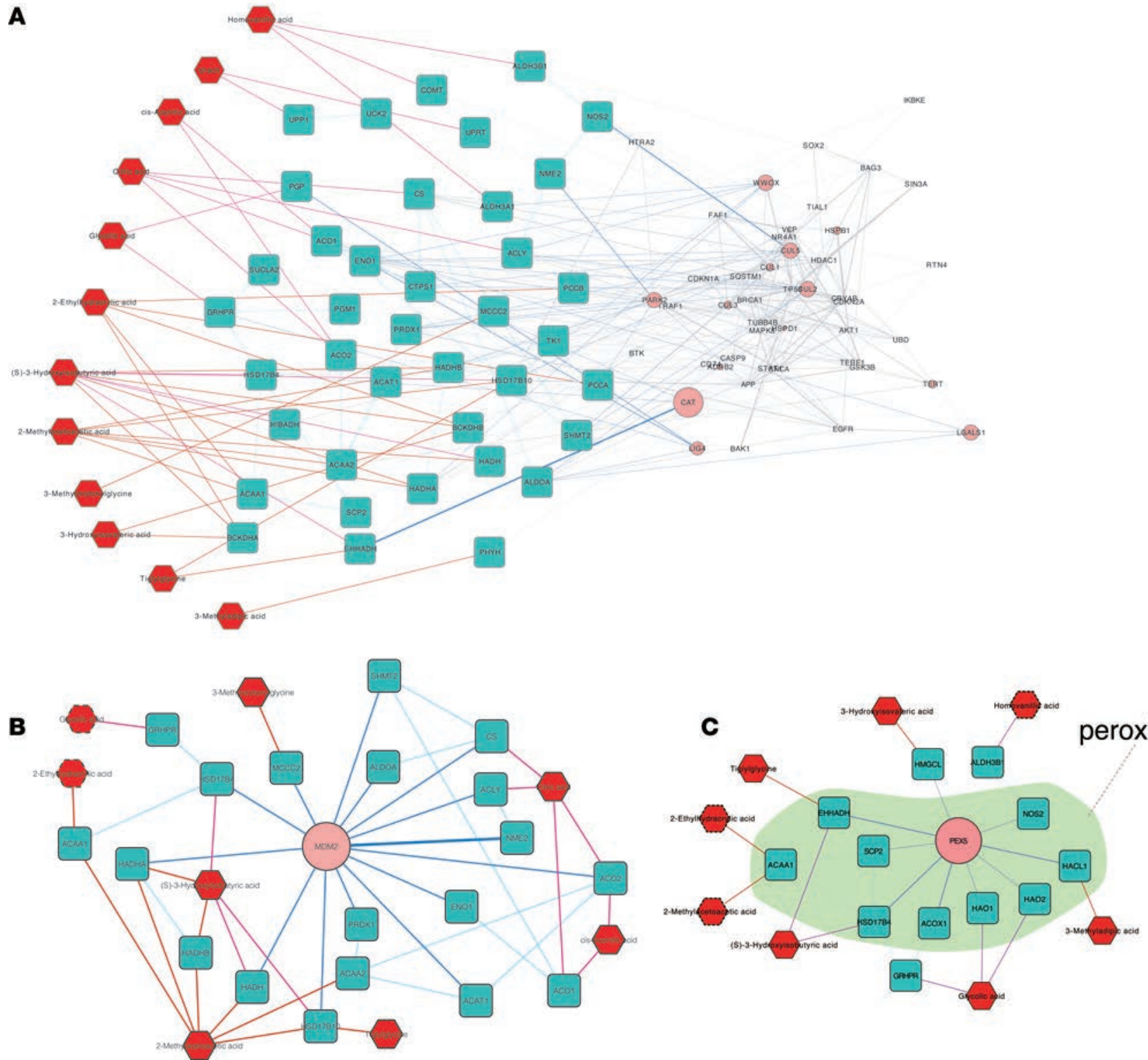

Figure 2. Subnetworks connecting part of $\mathbf{1 3}$ metabolites significantly decreased in diabetic kidney disease samples. (A) Subnetwork showing enzymes (blue rectangles) associated with bridge proteins (pink circles) that are known to have a function in apoptosis, and the metabolites (red hexagons) associated with these enzymes. (B) Subnetwork showing enzymes that interact with MDM2 (bridge protein) and metabolites that are associated with these enzymes. Six metabolites (solid red hexagons) are associated with the enzymes that directly interact with MDM2. Two metabolites (dotted red hexagons) are associated with the enzymes that interact with MDM2-interacting enzymes. (C) Subnetwork showing enzymes that interact with PEX5 (bridge protein) and metabolites that are associated with these enzymes. Peroxisomal components are marked in green. Five metabolites (solid red hexagons) are associated with the enzymes that directly interact with PEX5. Three metabolites (dotted red hexagons) are associated with the enzymes that interact with PEX5-interacting enzymes.

sponding to MDM2, HUWE1, PEX5, and $C U L 4 B, 4$ of the top 5 bridge proteins with the highest number of significant connections to the enzymes.

To confirm the biologic relevance of these enzymes, we analyzed the enriched functions of enzymes associated with the significant metabolites in the network using Cytoscape App BiNGO (15). Our results showed that there were 19 enriched biological processes (gene ontology [GO] terms, Supplemental Figure 2A) and 16 enriched cellular components (Supplemental Figure 2B) under the default threshold of $P$ values corrected for multiple testing. As further supportive evidence that our MetBridge ${ }_{\mathrm{DKD}}$ network reflects relevant pathways involved in diabetic kidney disease, we identified that citrate metabolic process genes and branched-chain amino acid metabolism were prominent in MetBridge ${ }_{\mathrm{DKD}}$, which had also been described in our previous 
Table 1. List of bridge proteins having a significant number of interactions with the enzymes associated with our 13 metabolites

\begin{tabular}{|c|c|c|c|c|}
\hline Gene Symbol & Gene annotation & $\begin{array}{l}\text { Number of } \\
\text { interacting enzymes }\end{array}$ & $\begin{array}{l}\text { Bonferroni-corrected } \\
\qquad P \text { value }\end{array}$ & Directly associated significant metabolite names \\
\hline MDM2 & $\begin{array}{l}\text { MDM2 proto-oncogene, E3 } \\
\text { ubiquitin protein ligase }\end{array}$ & 14 & 0.010287 & $\begin{array}{c}\text { (S)-3-Hydroxyisobutyric acid; cis-Aconitic acid; } \\
\text { Citric acid; 3-Methylcrotonylglycine; Tiglylglycine; } \\
\text { 2-Methylacetoacetic acid }\end{array}$ \\
\hline HUWE1 & $\begin{array}{l}\text { HECT, UBA and WWE domain- } \\
\text { containing } 1, \text { E3 ubiquitin } \\
\text { protein ligase }\end{array}$ & 12 & 0.003133 & $\begin{array}{c}\text { (S)-3-Hydroxyisobutyric acid; Citric acid; } \\
\text { 2-Ethylhydracrylic acid; 3-Methylcrotonylglycine; } \\
\text { Tiglylglycine; 2-Methylacetoacetic acid }\end{array}$ \\
\hline CUL4B & cullin 4B & 6 & 0.040394 & $\begin{array}{c}\text { (S)-3-Hydroxyisobutyric acid; 3-Methylcrotonylglycine; } \\
\text { Tiglylglycine; 2-Methylacetoacetic acid }\end{array}$ \\
\hline IFIT1 & $\begin{array}{l}\text { interferon-induced protein with } \\
\text { tetratricopeptide repeats } 1\end{array}$ & 4 & 0.025262 & 2-Ethylhydracrylic acid; 3-Methylcrotonylglycine \\
\hline
\end{tabular}

report (3) and by independent investigators $(16,17)$ (Supplemental Figure 2, A and B).

An important contribution of MetBridge is the identification of bridge proteins that connect relevant enzymes. To investigate whether functions of these bridge proteins are linked to relevant functional pathways, we evaluated the biological processes and cellular components of the bridge proteins (Supplemental Figure 2, C and D). We found that $22 \mathrm{GO}$ biological processes and $21 \mathrm{GO}$ cellular components were enriched. Forty-five proteins $\left(\sim 15.5 \%\right.$ of all proteins in MetBridge $\left._{\text {DKD }}\right)$ in the subnetwork had GO annotation of "regulation of apoptosis", which was identified as a significantly enriched GO term (Benjamini-Hochberg corrected $P$ value $<0.0484$ ). Previous studies have shown that high glucose induces apoptosis in various cells including podocytes and tubular epithelial cells, which both contribute to development of diabetic kidney disease (18). The subnetwork of MetBridge ${ }_{\mathrm{DKD}}$, which consists of bridge proteins involved in regulation of apoptosis, their interacting enzymes and metabolites associated with them, are shown in Figure 2A. We identified that 12 out of the 13 metabolites were connected by bridge proteins involved in apoptosis via the enzymes, indicating a strong relationship between apoptosis and the significantly regulated metabolites. Among the identified bridge proteins, we found cyclin-dependent kinases and inhibitors including $C D K N 1 A(p 21)$. $C D K N 1 A$ has been found to be consistently dysregulated in diabetic kidney and nondiabetic glomerular disease and is a well-known regulator of the cell cycle and apoptosis (19), or diabetic kidney disease (20). Therefore, there appears to be robust biological relevance of the identified bridge proteins.

Exploration of bridge proteins and enzymes connecting the significant metabolites on the network. We further focused on bridge proteins that have many interactions with the enzymes regulating the panel of 13 metabolites (hub bridge proteins), since such hub proteins are likely to have biologically important functions $(21,22)$. In particular, for each bridge protein, we calculated the statistical significance of the number of interactions with the set of enzymes associated with the 13 metabolites compared with the number of interactions with other enzymes in the global metabolic map of KEGG. We assumed that if an enzyme E1 is involved in metabolism of a metabolite $\mathrm{M}$ and another enzyme $\mathrm{E} 2$ interacts with $\mathrm{E} 1$, then $\mathrm{E} 2$ is associated with metabolite $\mathrm{M}$ and that the E1-E2 enzymatic unit has an effect on metabolism of $\mathrm{M}$ (e.g., interaction between the 2 enzymes $A C A A 1$ and SCP2 in Figure 2C).

The list of significant bridge proteins in the MetBridge ${ }_{\mathrm{DKD}}$ network was sorted by the number of interactions with the enzymes and is listed in Table 1 (the full list is given in Supplemental Table 1). Such significances are reflected by the sizes of the bridge proteins on the network shown in Cytoscape (Figure 1).

MDM2, HUWE1, PEX5, CUL4B, and IFIT1 were found to be the top 5 bridge proteins. Ubiquitin C (UBC) had the largest number of interactions (48) with the enzymes associated with our 13 metabolites; however, ubiquitin interactions with proteins are relatively nonspecific and it was not further evaluated. Nevertheless, the ubiquitin-proteasome system is known to be upregulated in the muscle of kidney disease patients and diabetic patients (23), and may indicate relevance of ubiquitin $C$ in patients with diabetic kidney disease based on the 
A

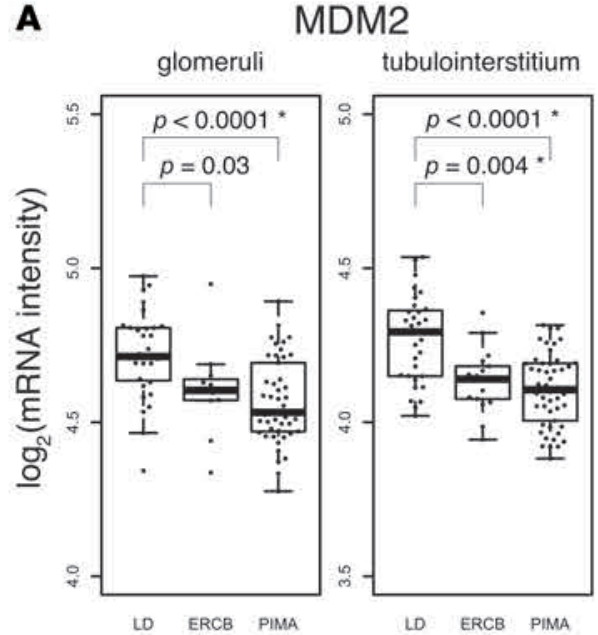

D

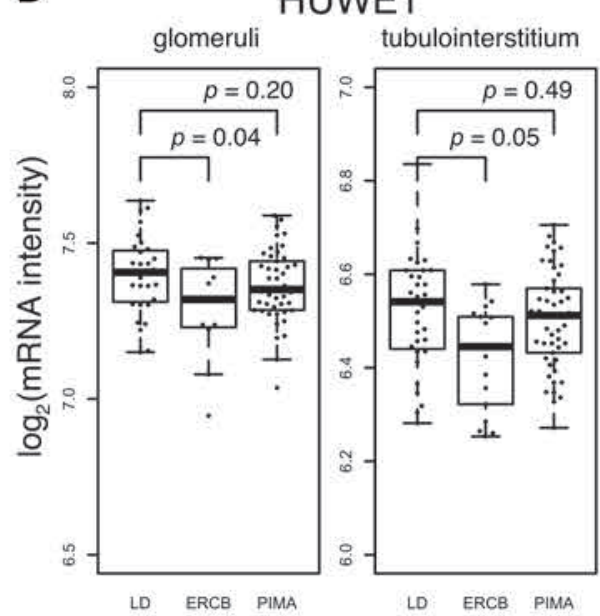

B

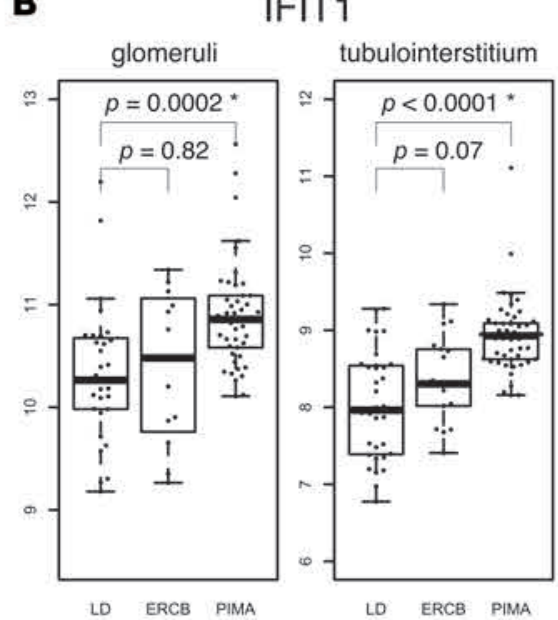

E

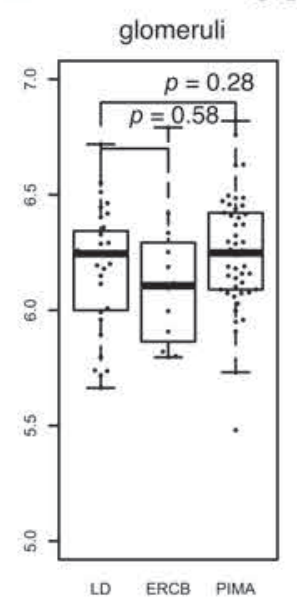

IFIT1

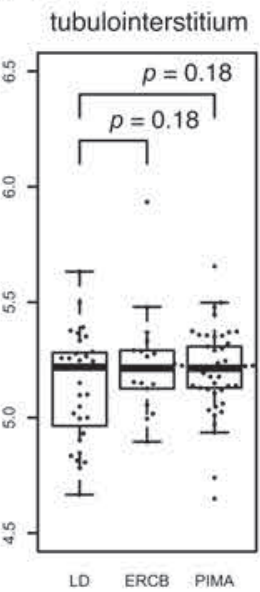

C

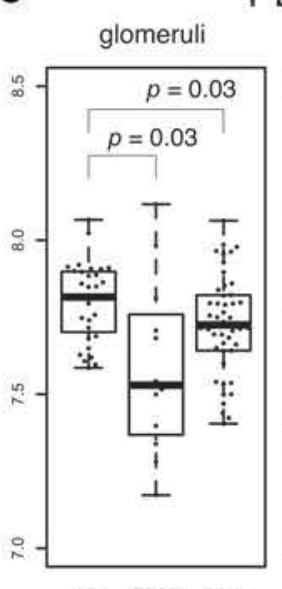

PEX5

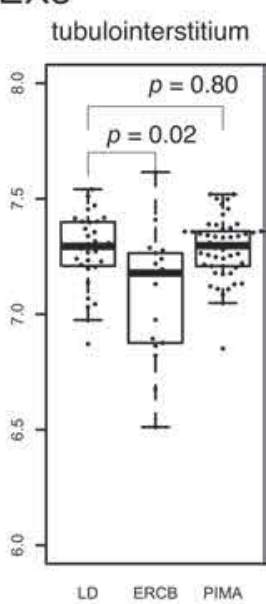

$\mathbf{F}$

TP53

tubulointerstitium
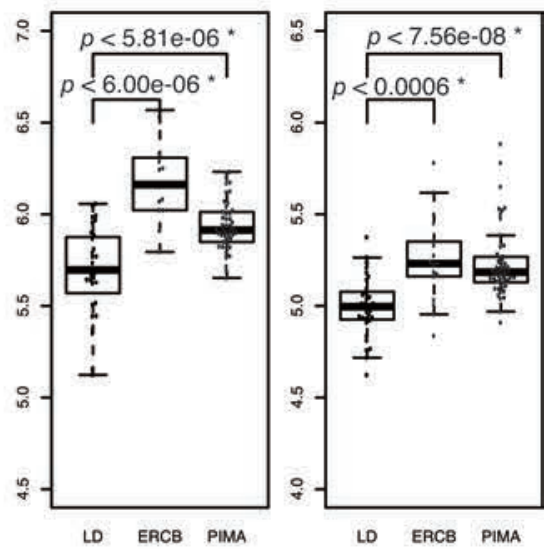

G

\section{Normal
kidney}

Diabetic kidney

\section{MDM2}

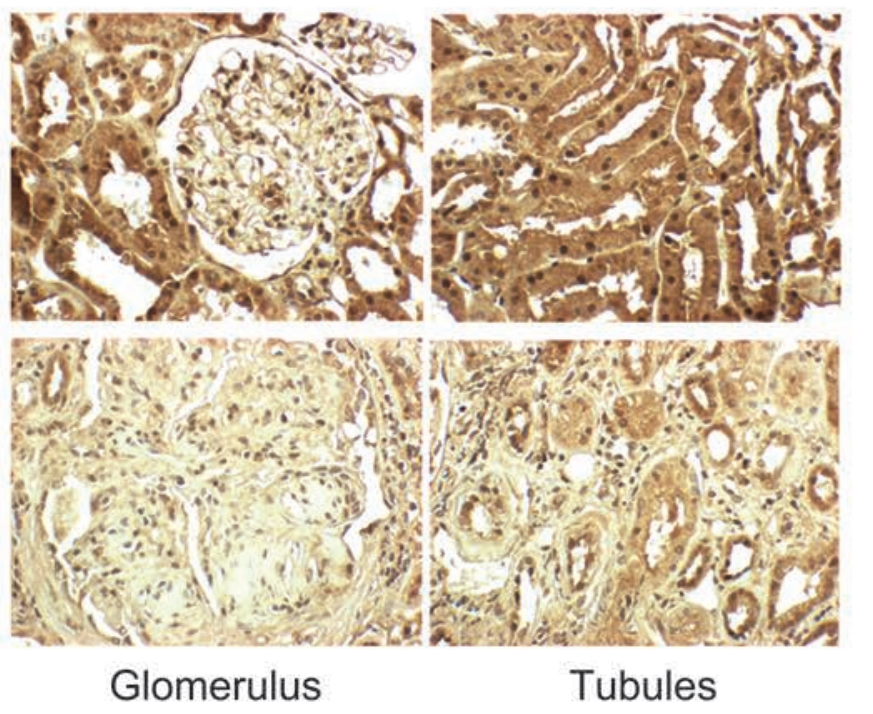

Figure 3. Expression levels of MDM2, HUWE1, PEX5, CUL4B, IFIT1, and TP53 in 2 independent data sets. Data from European Renal cDNA Bank (ERCB) and Native Americans study (Pima) were used for the gene expression analyses. LD is control (living donor). (A) Expression data for MDM2. (B-E) Gene expression data from top bridge proteins (IFIT1, PEX5, HUWE1, and CUL4B) that came out of our network analysis are shown. Shown $P$ values are uncorrected and based on $t$ tests. For the top 5 bridge proteins, the $P$ values that are significant $(<0.05)$ after Bonferroni correction for multiple testing are marked with asterisks $\left.{ }^{*}\right)$. (F) Expression data for TP53. The $P$ values were significant $(<0.05)$ after correction for multiple testing. Lower and upper edges of each box represents first and third quartiles of the distribution, respectively. The horizontal line in each box represents the median. The whiskers extend to $1.5 \times$ (third quartile - first quartile). (G) Representative immunostaining of MDM2 in normal human kidney biopsy tissue (representative from $n=2$ ) in upper panel and from a diabetic nephropathy biopsy tissue (representative from $n=3$ ) in lower panel. Magnification, $\times 40$. 
A
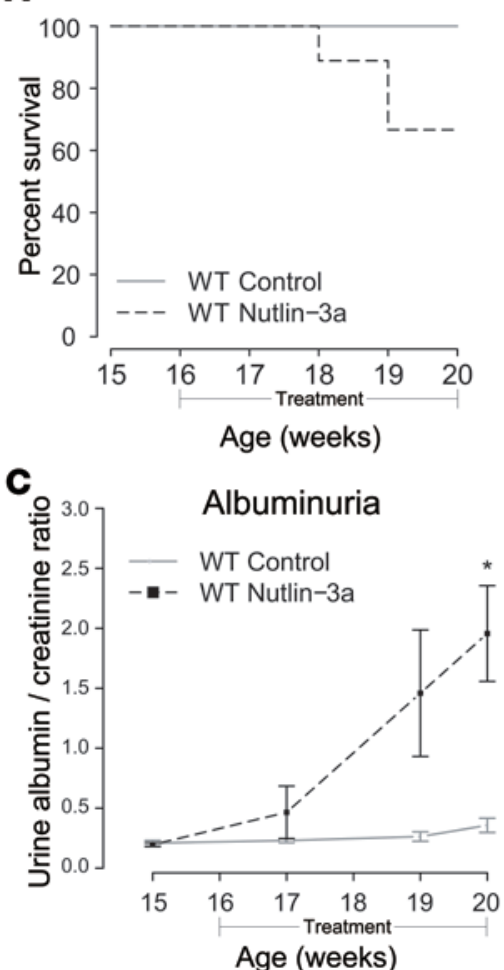

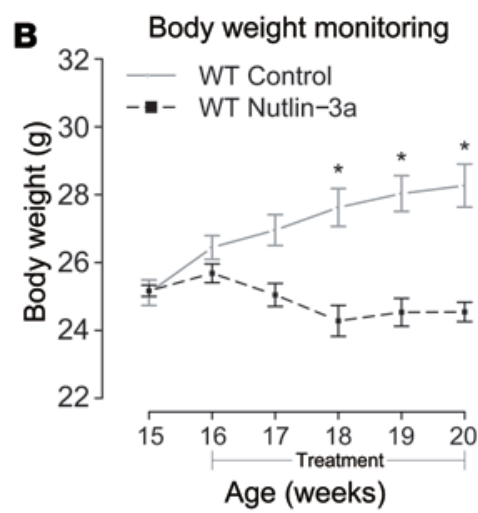

D Blood urea nitrogen at 20 weeks

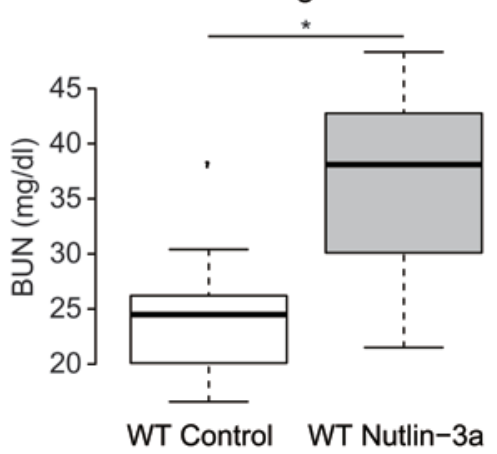

Figure 4. Pharmacological inhibition of MDM2 in C57B6/JL healthy mice affects glomerular filtration barrier and kidney function. (A) Survival of Nutlin-3a-treated mice (66\% at the end of the study) compared with that of control mice (100\%, $P=0.19$, log-rank test). (B) Body weight monitoring along the treatment. While control mice increased progressively in weight, Nutlin3a-treated mice lost weight progressively during the treatment, being significant from 18 weeks onwards compared with control mice. ${ }^{*} P<0.001$, $t$ test. Control, $n=9$; Nutlin-3a, $n=9$. (C) Albuminuria measured as urine albumin/creatinine ratio (ACR) along the study. ACR remained normal for control mice but increased progressively in Nutlin-3a-treated mice, being significantly higher at the end of the study. ${ }^{*} P<0.05$, 1-tailed $t$ test. Control, $n=7$; Nutlin-3a treated, $n=7$. (D) Blood urea nitrogen (BUN) levels at the end of the study were significantly higher in Nutlin-3a-treated mice compared with control mice. ${ }^{*} P<0.05, t$ test. Control group, $n=9$; Nutlin-3a group, $n=$ 7. Error bars represent standard errors (B and $\mathbf{C}$ ). For box-and-whisker plots, the horizontal line in each box represents the median (D). The whiskers extend to $1.5 \times$ (third quartile - first quartile).

urine metabolite profile. Indeed, among the top 5 bridge proteins, 3 proteins, MDM2, HUWE1, and $C U L A B$ have been linked to ubiquitination.

MDM2 bridges 6 metabolites by directly interacting with 14 enzymes in the network (Figure 2B and Table 1) and bridged 2 metabolites indirectly (glycolic and 2-ethylhydracrylic acid). Citric acid was associated with 9 enzymes (4 directly associated with citric acid and 5 indirectly associated through these 4 enzymes), 8 of which interacted with MDM2. Additionally, 2-methylacetoacetic acid and cis-aconitic acid also had significant numbers of associations with MDM2. MDM2 also bridged 3-methylcrotonylglycine, tiglylglycine (with 1 enzyme each), and 3-hydroxyisobutyric acid.

$P E X 5$ is a peroxisomal biogenesis marker essential for the assembly of functional peroxisomes involved

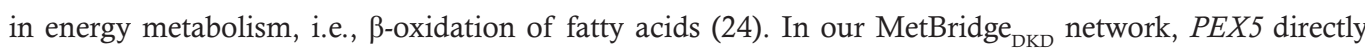
interacts with 9 enzymes, 6 of which are directly associated with 5 metabolites (Figure 2C), 3 of which are related to fatty acid metabolism (i.e. tiglylglycine, 3-hydroxyisovaleric acid, and 3-methyladipic acid). Enzymes interacting with PEX5 were linked to 3 additional metabolites through other enzymes. To determine which metabolite had the most significant number of associations with the set of enzymes that are enriched with PEX5-interacting partners, we assessed for metabolite overlap between enzymes associated with the metabolite and those which interact with PEX5. We found that glycolic acid had the strongest association with PEX5-interacting enzymes.

Interferon-induced protein with tetratricopeptide repeats 1 (IFIT1) interacted with 4 enzymes (MCCC1, $M C C C 2, P C C A$, and $P C C B$ ), which are associated with 2 metabolites, 2-ethylhydracrylic acid and 3-methylcrotonylglycine (Table 1). HUWE1 (HECT, UBA, and WWE domain-containing 1, E3 ubiquitin protein ligase) and cullin 4B (CUL4B) interacted with 12 and 6 enzymes, respectively, which are associated with 6 and 4 metabolites, respectively. The brief descriptions of these enzymes and metabolites are provided in Supplemental Table 1.

Gene expression changes of bridge proteins in independent cohorts. As an independent validation regarding the possible relevance of the identified bridge proteins to human diabetic nephropathy, we investigated gene expression levels of the top hub bridge proteins in patients with diabetic kidney disease from 2 independent study cohorts, the European Renal cDNA Bank (ERCB) human diabetic kidney disease and Native Americans from the Gila River Indian Community in Arizona (Pima). Gene expression levels in glomeruli and tubulointerstitium of patients with kidney disease were compared with those of controls (living donors).

We found that the gene expression level of $M D M 2$ was decreased in glomeruli and tubulointerstitium of patients with kidney disease in the ERCB and the Native American data set (Figure 3A). To evalu- 
A

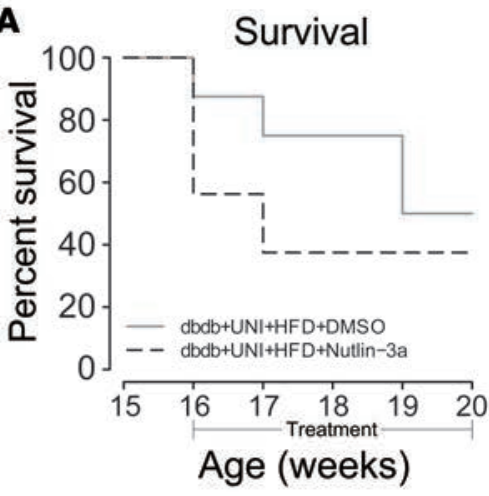

B

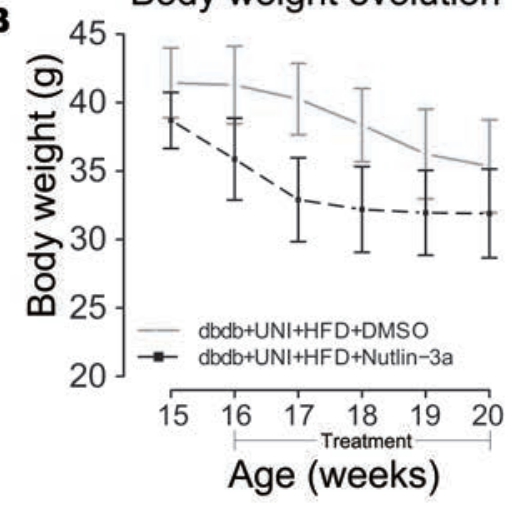

C Blood urea nitrogen

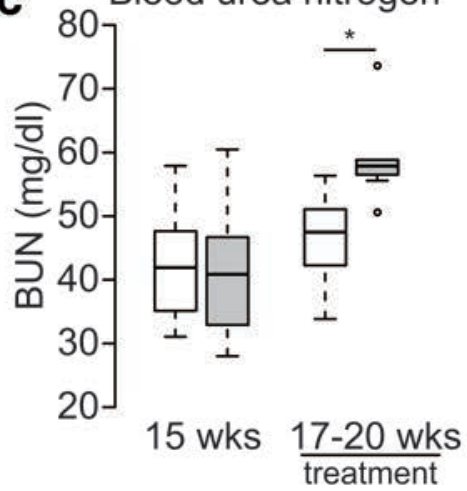

D
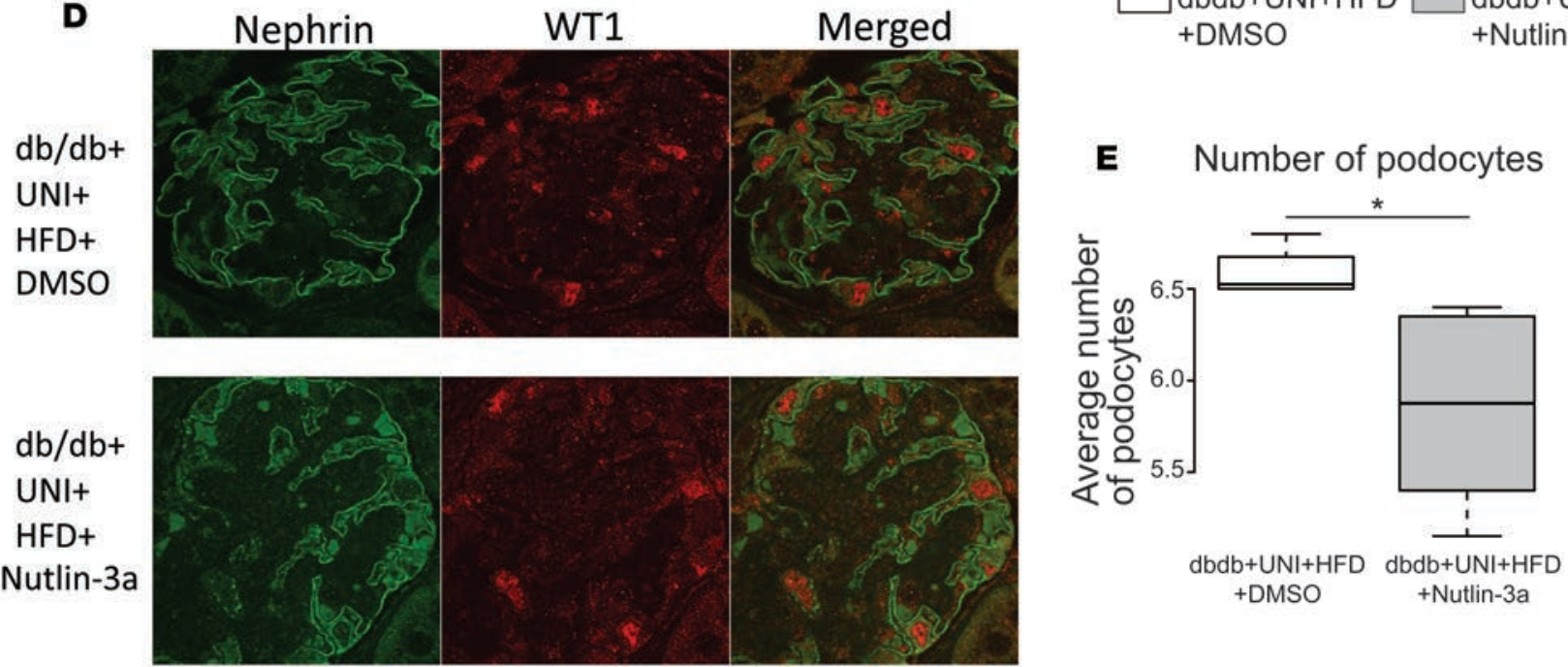

$\mathbf{F}$

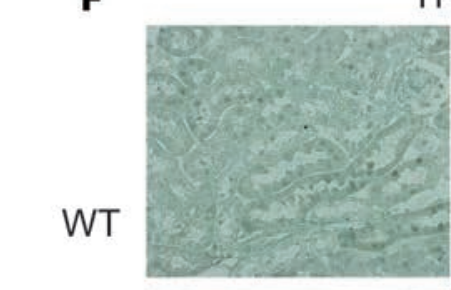

$\mathrm{db} / \mathrm{db}$

Vehicle

TP53
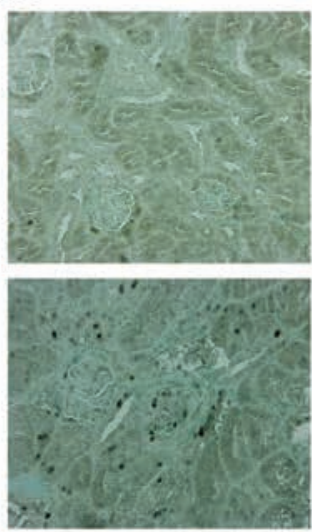

Nutlin-3a
Figure 5. Pharmacological inhibition of MDM2 in uninephrectomized Leprdb-/(db/db) mice fed with high-fat diet. (A) Survival in Nutlin-3a-treated db/db mice (37.5\% at the end of the treatment) compared with that of vehicle-treated $\mathrm{db} / \mathrm{db}$ mice ( $57 \%, P=0.2$, log-rank test). (B) Body weight monitoring along the treatment. Both groups lost weight during the treatment, though Nutlin-3a-treated mice had a lower body weight compared with vehicle-treated mice. (C) Blood urea nitrogen (BUN) levels over the treatment increased in both groups, being higher in Nutlin-3a-treated mice than in vehicle-treated mice. ${ }^{*} P=0.0039, t$ test, $n=7 /$ group. (D) Immunofluorescence (IF) image of control mouse in upper panel and of Nutlin-3a-treated mouse in lower panel. Magnification, $\times 60$. (E) IF quantification of number of podocytes. Nutlin-3a-treated displayed a lower number of podocytes $(6.50 \pm 0.14)$ than vehicletreated mice $(5.84 \pm 0.54)$. ${ }^{*} P=0.0139$, Wilcoxon rank sum test. $\mathrm{db} / \mathrm{db}$ DMSO, $n=$ 4; $\mathrm{db} / \mathrm{db}$ Nutlin-3a, $n=6$. (F) Representative photomicrograph of TP53 staining in the wild-type and $\mathrm{db} / \mathrm{db}$ mice. Nutlin-3a treatment in $\mathrm{db} / \mathrm{db}$ mice causes upregulation and nuclear shift of TP53 in renal cells. Representative from $n=5$ for each group except wild type treated with Nutlin-3a $(n=4)$. Magnification, $\times 200$. Error bars in B represent SE. For box-and-whisker plots in $\mathbf{C}$ and $\mathbf{E}$, the horizontal line in each box represents the median. The whiskers extend to $1.5 \times$ (third quartile - first quartile).

ate $M D M 2$ protein levels, immunostaining was performed in biopsy samples from patients with diabetic nephropathy and compared with control samples. As noted in Figure 3G, there was a marked reduction in both glomerular and tubulointerstitial staining of $M D M 2$ in the diabetic samples.

Increases in IFIT1 expression levels were observed in the Native American group but not in the ERCB group (Figure $3 \mathrm{~B}$ ). We also found that PEX5 was decreased in the ERCB and Native Americans groups (Figure 3C). HUWE1 showed a reduction in the ERCB group but not in the Native American group (Figure 3D). No significant difference was observed for CULAB in any of the groups (Figure 3E). Therefore, 
PAS

A
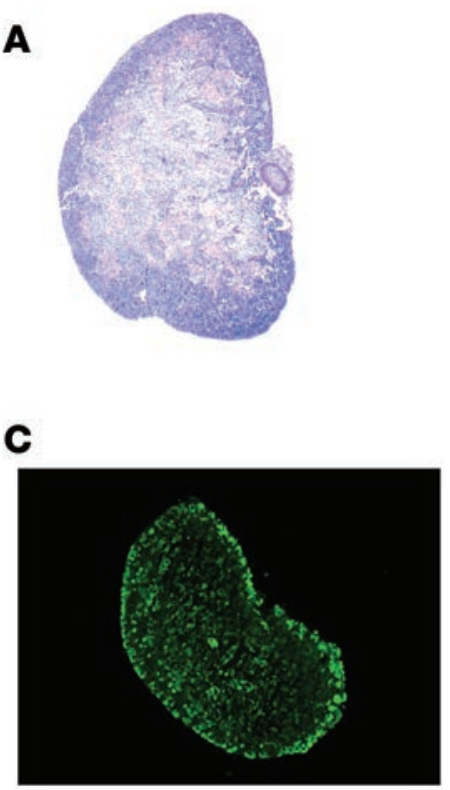

Control
B

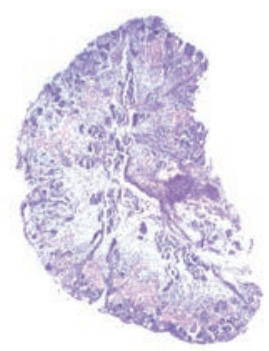

D

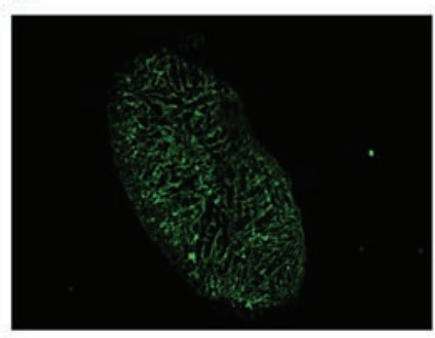

Nutlin-3a
Figure 6. Effect on renal development and WT1 expression in a 17.5-day embryonic kidney in response to Nutlin-3a treatment. Periodic acid-Schiff (PAS) staining of kidney sections ( $\mathbf{A}$ and $\mathbf{B}$ ) and immunofluorescent staining using polyclonal antibody against WT1 (green, $\mathbf{C}$ and $\mathbf{D}$ ) in a 17.5-day embryonic kidney treated with $5 \mu \mathrm{M}$ Nutlin-3a or DMSO for 5 days. Representative from $n=3$ /group. Magnification, $\times 10$.

gene expression levels of 4 out of 5 top bridge proteins were altered to various degrees with independent transcriptomic analysis of human diabetic nephropathy tissue samples. Of these, MDM2 had the most consistent downregulation in both glomerular and tubulointerstitial compartments in 2 separate clinical cohorts.

Pharmacological inhibition of MDM2 function affects podocyte integrity in heathy mice and worsens diabetic kidney disease in a diabetic mouse model. As gene expression level of $M D M 2$ was consistently downregulated in multiple cohorts of patients with diabetic nephropathy and in both glomerular and tubulointerstitial compartments, we evaluated the functional role of $M D M 2$ in kidney dis-

ease. $M D M 2$ is a ubiquitin E3 ligase and can shuttle between the cytoplasm and the nucleus (25). One of the best-known cellular functions of MDM2 is to bind to the tumor suppressor TP53, which enhances TP53 ubiquitination and targets it for proteasomal degradation (26). A possible physiological role for $M D M 2$ and TP53 in glomerulosclerosis has been previously described (27). Interestingly, we found that the expression level of TP53 was increased in the 2 patient cohorts of diabetic kidney disease (Figure 3F).

The effect of inhibiting MDM2 function with Nutlin-3a was evaluated in healthy and diabetic mice. Nutlin-3a is a cis-imadozolin compound that functions as an MDM2 antagonist by occupying the MDM2 TP53-binding pocket and inhibiting the TP53 pathway with a high degree of specificity (28-31). Nutlin-3a was injected intraperitoneally on alternate days during 4 weeks in 16 -week-old C57BL/6 healthy mice. A vehicle group was injected with the solvent, dimethyl sulfoxide (DMSO), and a control group was kept in the same animal facility without receiving any intervention. The latter 2 groups presented no differences in any of the analyses and were treated as a combined control group. Surprisingly, Nutlin-3a-treated mice had increased mortality (Figure 4A), with reduced body weight (Figure 4B) and an increase in albuminuria (measured as the albumin/creatinine ratio [ACR]) (Figure 4C) and plasma urea levels (blood urea nitrogen [BUN]) (Figure 4D), compared with control. This result demonstrated that $M D M 2$ inhibition was sufficient to induce kidney dysfunction and increase mortality in wild-type mice.

The role of $M D M 2$ was then evaluated in $L_{e p r d b}^{-/-}(\mathrm{db} / \mathrm{db})$ mice. This model of diabetic kidney disease has modest glomerular changes and minimal tubulointerstitial changes with diabetes. MDM2 immunostaining did not show differences in the $\mathrm{db} / \mathrm{db}$ versus nondiabetic heterozygous mice $(\mathrm{db} / \mathrm{m})$ in the glomerular and tubulointerstitial compartments (Supplemental Figure 3). To inhibit MDM2, mice were injected with either Nutlin-3a or DMSO on alternate days during 4 weeks. To stress the phenotype, all the mice underwent uninephrectomy at 7 weeks of age and were fed a high-fat diet. Overall mortality was very high in both groups, but more prominent in the Nutlin-3a-treated group (Figure 5A). Although there was a trend toward lower body weight in Nutlin-3a-treated mice, no statistically significant difference was observed in body weight (Figure 5B) or in glycemia between the 2 groups. However, as seen in control mice treated with Nutlin-3a, the diabetic mice treated with Nutlin-3a had increased BUN in association with increased mortality (Figure 5C). Additionally, immunostaining of kidney sections for WT1 and nephrin showed a significantly lower number of podocytes in Nutlin-3a-treated compared with vehicle-treated mice (Figure 5, D and E). Expression levels of podocyte genes were uniformly reduced in Nutlin-3a-treated diabetic mice (Supplemental Figure 4, A-F). There was also increased immunostaining of TP53 in the kidneys from mice treated with Nutlin-3a compared with the controls for both wild-type and $\mathrm{db} / \mathrm{db}$ mice (Figure $5 \mathrm{~F}$ ). However, there was an increase in TP53-positive nuclei in $\mathrm{db} / \mathrm{db}$ mice treated with Nutlin-3a. 


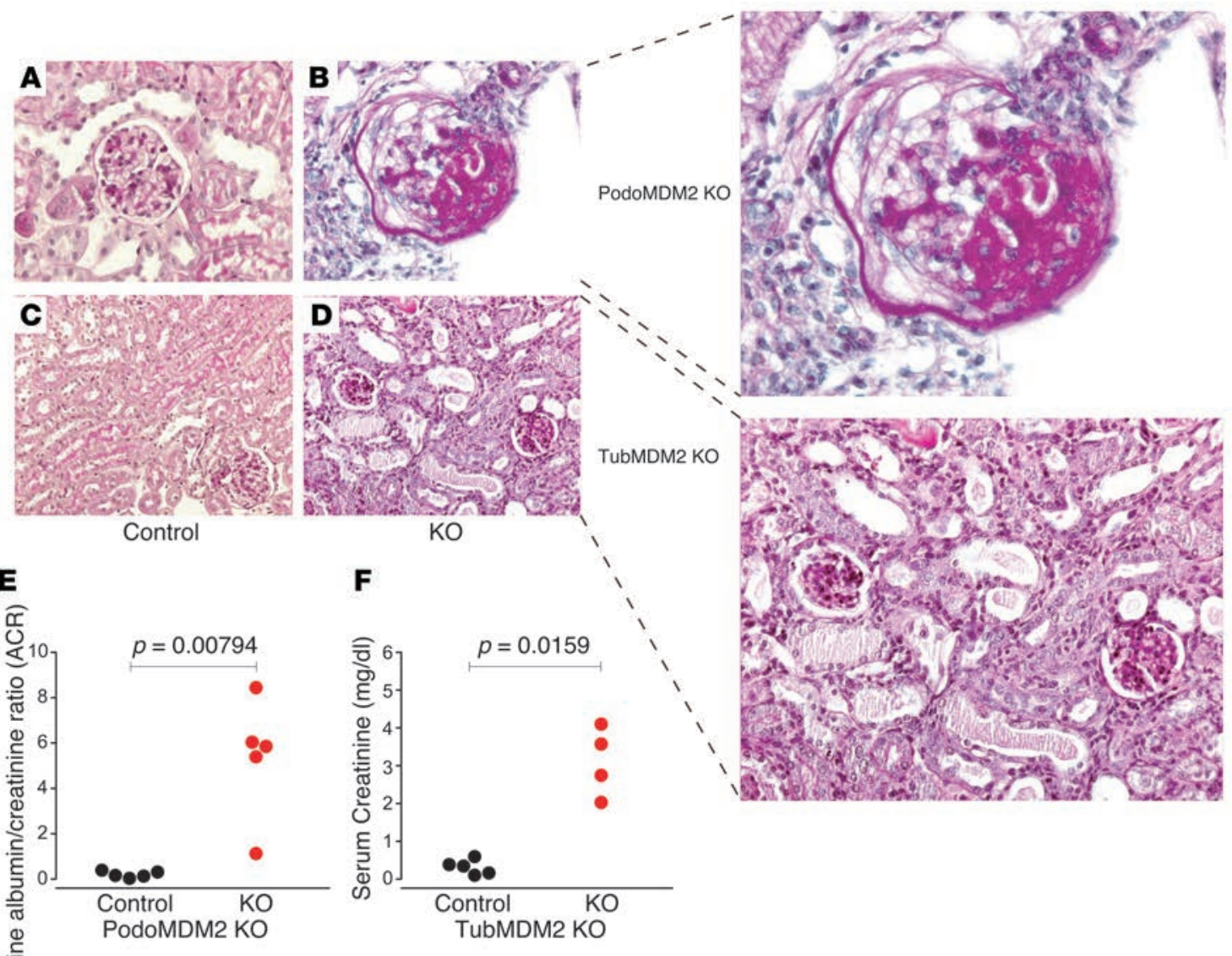

G 3-Methylcrotonylglycine adjusted combined $p=0.005$

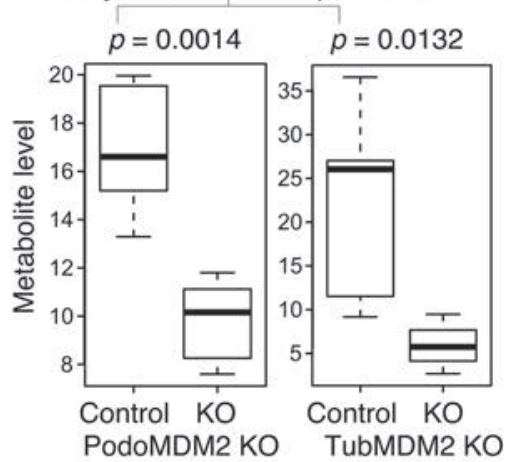

H

Uracil

adjusted combined $p=0.030$

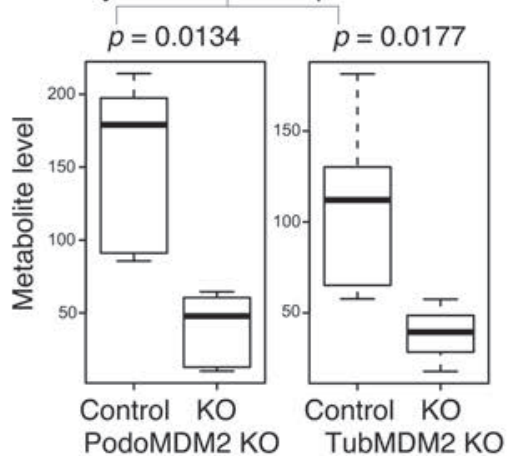

Figure 7. Pathophysiological analyses of MDM2-knockout mice. Representative photomicrographs from (A) control, (B) the podocyte-specific MDM2-knockout mouse (PodoMDM2), (C) control, and (D) the tubule-specific MDM2-knockout mouse (TubMDM2KO) subcapsular cortical area. Images B-D are magnified on the right. Representative image (B) shows a focal glomerular sclerosis and a partial glomerular collapse in the periodic acid-Schiff-stained (PAS-stained) kidney sections of the podocyte-specific MDM2-knockout mouse. The representative PAS image (D) depicts the tubular damage in the form of tubular vacuolization, tubular casts, and tubular atrophy in the renal sections of the tubule-specific MDM2-knockout

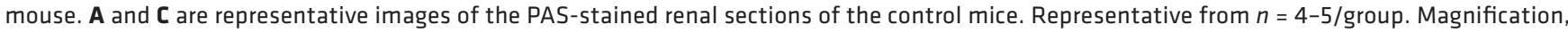
$\times 400$ (A and B) and $\times 200$ (C and D), further magnified by 1.75 (B and D) using Adobe Illustrator. (E and F) Pathophysiological analyses of control and knockout mice. (E) Urine albumin/creatinine ratio of podocyte-specific MDM2-knockout mice compared with controls. (F) Serum creatinine (mg/dl) of tubule-specific MDM2-knockout mice versus control group. Shown $P$ values are based on Mann-Whitney $U$ test. Metabolite levels of (G) 3-methylcrotonyl glycine and $(\mathbf{H})$ uracil in control and MDM2-knockout mice. Distinct control samples for podocyte-specific and tubule-specific mice were used. $P$ values were calculated based on log2 (metabolite level) ( $t$ test). The pair of $P$ values for podocyte- and tubule-specific knockout mice for each metabolite was combined using Fisher's method and the combined $P$ values were corrected for multiple testing using Benjamini-Hochberg, $s$ method. For box-and-whisker plots, the horizontal line in each box represents the median ( $\mathbf{G}$ and $\mathbf{H}$ ). The whiskers extend to $1.5 \times$ (third quartile - first quartile). 
MDM2 inhibition in mouse embryonic kidney development. MDM2 has ubiquitous expression throughout the embryo and may play an important developmental role $(32,33)$. To determine if $M D M 2$ inhibition may have an effect on embryonic kidney development, Nutlin-3a was administered to embryonic kidneys. The Nutlin-3a-treated embryonic kidney sections (17.5 days) showed that the nephrogenic zone was poorly developed (Figure 6, A and B). The Nutlin-3a-treated embryonic kidney sections also showed reduced expression of WT1 via immunofluorescent staining (Figure 6, C and D) compared with DMSO-treated control embryonic kidneys.

Pathophysiological and metabolomics analyses of MDM2-knockout mice. We also conducted experiments with $M D M 2$-knockout mice to further investigate cell-specific roles of $M D M 2$. There was a marked degree of glomerular expansion and severe glomerular sclerosis in podocyte-specific MDM2-knockout mice (Figure 7, A and B), whereas in the tubule-specific $M D M 2$-knockout mouse there was tubular dilatation in addition to tubular atrophy and tubulointerstitial fibrosis (Figure 7, C and D). There was also a marked increase in the urine ACR in the podocyte-specific MDM2-knockout mice (Figure 7E), and an increase in serum creatinine in the tubule-specific $M D M 2$-knockout mice (Figure 7F).

Metabolomic analysis of the urine from MDM2-knockout mice revealed a significant reduction in urine levels of 3-methylcrotonylglycine in the podocyte-specific MDM2-knockout mice and the tubule-specific MDM2-knockout mice (Figure 7G). Urine uracil was also significantly reduced in both the podocyte-specific and tubule-specific knockout mice (Figure 7H). The remaining urine metabolites from the panel of 13 metabolites previously found to be reduced in human diabetic kidney disease (3) were not different in the cell-specific MDM2-knockout mice as compared with wild-type controls.

\section{Discussion}

We developed a new software, MetBridge Generator, on Cytoscape to provide a useful way to analyze metabolomics data (Supplemental Figure 1). MetBridge Generator identified several key proteins that were significantly altered at the gene-expression level in kidney biopsy tissue from independent cohorts of patients with diabetic nephropathy (Figures 2 and 3, and Table 1). The App alone cannot predict whether the key proteins have a causal role; however, new hypotheses can be developed based on the possible cellular or physiological role of the key proteins. Of the proteins that were identified from the MetBridge Generator network analysis, we found that $M D M 2$ was consistently downregulated by gene expression analysis in patients with diabetic nephropathy (Figure 3A) and plays a key role in progressive diabetic kidney disease. Interestingly, reduced $M D M 2$ in podocytes or in tubular cells was associated with enhanced renal disease and reduction in 2 key metabolites, 3-methylcrotonylglycine and uracil (Figure 7, G and H). These 2 metabolites were part of the panel of metabolites that were consistently reduced in patients with diabetic kidney disease (3).

Using MetBridge Generator allowed the identification of relevant enzymes and bridge proteins that link human metabolomics data to the pathophysiology of diabetic kidney disease at a molecular level. The enrichment of enzymes participating in citrate metabolic process and branched-chain amino acid metabolism from our MetBridge ${ }_{\mathrm{DKD}}$ network (Supplemental Figure 2A) confirmed the role of these pathways in diabetic kidney disease. Linkage of these functions to diabetic kidney disease was supported not only by our recent study (3) but also by independent studies in mouse models (17), and in diabetic patients (34), which further support the relevance of our systems approach. Our approach also supports the value of identifying key proteins/metabolites that are implicated via guilt by association; i.e., interacting proteins/ metabolites tend to share similar cellular functions and may function in pathogenesis (35).

We further focused on hub bridge proteins that have a significant number of interactions with enzymes associated with our 13 metabolites, as hubs in biological networks tend to have a large impact on cellular processes. MDM2 emerged as the most relevant protein based not only on a large number of interactions with the enzymes associated with our relevant metabolites (Figure $2 \mathrm{~B}$ ), but also on the significant reduction in $M D M 2$ gene expression in patients with diabetic kidney disease from independent cohorts (Figure $3 \mathrm{~A}$ ). As $M D M 2$ was consistently downregulated in both glomerular and tubulointerstitial compartments from human kidney biopsies, we suggest that MDM2 may play a key functional role in both compartments.

Our studies on $M D M 2$ in healthy and diabetic mouse models support a key role for $M D M 2$ in the pathophysiology of progressive kidney disease. The pharmacological inhibition of MDM2 in healthy mice with the antagonist Nutlin-3a showed a negative effect on kidney function in otherwise healthy mice, as seen by the significant increase in BUN levels and proteinuria (Figure 4, C and D). Since MDM2 expression in the kidney is mainly localized in tubules and podocytes (36), it is likely that the impairment in renal function 
is due to a combined disturbance in podocyte and tubular integrity as a result of $M D M 2$ inhibition. Prior studies have found that MDM2 reduction in mouse models exhibited impairment in podocyte homeostasis, caused by upregulation of TP53 and consequent cell death (27). Moreover, inhibition of MDM2 with Nutlin-3a in diabetic mice revealed an accelerated disease progression with rapid podocyte depletion (Figure 5, D and E). As TP53 was increased in the nuclei of diabetic mice treated with Nutlin-3a (Figure 5F), a key role for TP53 in mediating apoptosis and possible senescence in the diabetic kidney is likely. Of note, the $\mathrm{db} / \mathrm{db}$ diabetic mouse model does not develop progressive diabetic kidney disease, as MDM2 levels are not reduced under basal condition. The reduction in $M D M 2$ with Nutlin-3a is required to mimic the human diabetic kidney disease condition.

The present study also demonstrated that MDM2 deletion in podocytes enhanced glomerulosclerosis and urinary albumin (Figure 7, B and E). MDM2 deletion in tubular cells resulted in an intact glomerular structure but there was a marked tubulointerstitial injury pattern consistent with progressive chronic kidney disease (Figure 7D). The tubule-specific MDM2 knockout also exhibited an increase in serum creatinine (Figure 7F), further supporting the important role for tubular MDM2 in the progressive decline of renal function of diabetic kidney disease. The gene knockout studies support the important role that MDM2 likely has in human diabetic kidney disease, as both glomerular and tubulointerstitial levels of MDM2 gene expression were significantly reduced in cohorts of human diabetic nephropathy.

Interestingly, with urine metabolomics analysis from the $M D M 2$-knockout mice we found that 2 metabolites among the 13 metabolites identified in patients with diabetic kidney disease, 3-methylcrotonylglycine and uracil, were significantly decreased (Figure 7, G and $\mathrm{H}$ ). These were the only 2 metabolites that were reduced in both podocyte- and tubule-specific MDM2-knockout mice. 3-Methylcrotonylglycine has a connection with $M D M 2$ via methylcrotonoyl-CoA carboxylase 2 (MCCC2) (Figure 2B). Mutations in $M C C C 2$ lead to reduced catabolism of leucine, one of the branched-chain amino acids, and this leads to increased 3-methylcrotonylglycinuria (37). Notably, the expression level of MCCC2 was significantly decreased in glomeruli of the Native American cohort and tubules of the ERCB cohort (Supplemental Figure 5).

The metabolite uracil was also reduced in the MDM2-knockout mice and was previously identified in the signature for human diabetic kidney disease. Of interest, plasma pseudouridine was increased in patients with diabetes who progressed to end-stage renal disease compared with those who did not (38). Since pseudouridine is synthesized from uracil, increased pseudouridine synthesis may result in decreased uracil. Interestingly, pseudouridine synthase dyskerin ( $D K C 1)$ affects modification of uridine residues to produce pseudouridine on rRNA, and an increased level of nascent preribosomal RPL5/RPL11/5S rRNA complexes in response to impaired ribosome biogenesis inhibits the activity of MDM2 (39). Thus, one of the possible connections between uracil and $M D M 2$ under the disease condition may involve biogenesis and the modification pathway of rRNA. Although we focused on metabolic pathways and PPIs, this hypothesis suggests that incorporating protein-RNA interactions into our network may elucidate additional key pathways underlying disease progression.

We further found that inhibition of $M D M 2$ with Nutlin-3a in the embryonic kidney caused defects in development of the nephron (Figure 6), suggesting that MDM2 is important not only in the adult stage but also in the embryonic stage. Consistent with our results, knocking out MDM2 in mouse embryos and MDM2 knockdown by siRNA in podocytes of adult mice caused cell death $(27,32)$. A previous study in $M D M 2$-deficient mice also demonstrated that defects in kidney development at fetal stages enhance development of hypertensive and chronic kidney disease at the adult stage (40-42). It is conceivable that the kidney may undergo periods of regeneration during the natural history of diabetic nephropathy and regeneration and development of new tubular cells will be inhibited with MDM2 reduction. Reduction of $M D M 2$ will also predispose the diabetic kidney to impaired recovery from bouts of acute kidney injury.

We also found that $P E X 5$ may also contribute to diabetic kidney disease via network analysis and gene expression studies (Figures $2 \mathrm{C}$ and $3 \mathrm{C}$ ). PEX5 is one of the proteins essential for the assembly of functional peroxisomes and plays an essential role in peroxisomal protein import. Defects in this gene have been associated with Zellweger syndrome (43). Peroxisomal dysfunction by catalase deficiency in diabetic mice has been associated with reduction in PEX5 expression levels, impaired peroxisomal and mitochondrial biogenesis, and accelerated diabetic renal disease and fibrosis (44). Thus, PEX5 is associated with diabetic nephropathy via peroxisomal dysfunction and we suspect that it may play a role in the significant downregulation of peroxisome-related metabolites. 
In conclusion, we present an App that allows for efficient focused analysis of urine metabolomics data from patients with diabetic kidney disease. The App will also be relevant to the interpretation of many metabolomic signatures from a variety of diseases. The present version of the application has revealed a potentially novel role for the key protein $M D M 2$ in human diabetic kidney disease. The role of $M D M 2$ was supported by gene expression analysis in human diabetic nephropathy and with pharmacologic and genetic reduction of $M D M 2$. Reduction of $M D M 2$ likely plays a key role to enhance diabetic glomerular and tubular disease. Currently, only a fraction of mammalian cellular metabolic pathways are well annotated and accessible in publicly available databases. As our knowledge of metabolic pathways expands and is collated in publicly available databases, there will be new connections among metabolites leading to new target identification. Similar types of new bioinformatics tools will be critical to exploit metabolomics data to provide new disease insights.

\section{Methods}

\section{Data collection}

Metabolic pathway was obtained from the global human metabolic pathway map (http://www.genome. jp/kegg/, hsa01100.xml, creation date: Apr 10, 2015) stored in the KEGG database, which contains information about connections between metabolites through enzymes. We particularly used information about which metabolite is converted to which metabolite through a reaction catalyzed by which enzyme. A set of human PPI data was downloaded from the BioGRID database (version 3.3.123, http://thebiogrid.org). By converting enzymes participating in metabolic pathways into protein information, these 2 data sources were integrated. Metabolic pathways related to the 13-metabolite panel linked to diabetic kidney disease in our previous publication was also added (3). The final global network, which contains comprehensive metabolic pathways and PPI information, was designated as "MetBridge" network. Detailed information about gene and enzyme information can be obtained from NCBI Entrez Gene (gene annotation, etc.) and BRENDA, respectively. Information about metabolites (HMDB numbers, corresponding KEGG IDs, etc.) were obtained from Human Metabolite Database (HMDB version 3.5-3.6).

\section{Visualization by Cytoscape}

The integrated interaction network containing metabolites, enzymes, and bridge proteins was loaded onto Cytoscape (version 3.1.1 or 3.2.1), a software platform for network visualization and analyses. Nodes were colored differently for metabolites, enzymes, and bridge proteins. We also assigned different shapes for these 3 types of nodes. Edge widths of PPIs were proportional to the number of independent studies reporting the corresponding interactions so that a user can have some idea as to which interactions are reliable. The user can browse the network to get detailed information on each metabolite, enzyme, or bridge protein.

\section{Cytoscape App development}

The Cytoscape App equipped with simple graphical user interface was developed using programming language Java to find PPIs that connect significant metabolites within a step from enzymes associated with significant metabolites. Technically, the App searches for metabolites in the MetBridge and looks for their first and second neighbors, which correspond to enzymes and bridge proteins, respectively. Then the subnetwork is displayed as a separate window. The App is a jar file that can be installed using the Cytoscape Apps menu (tested on Cytoscape 3.1.1 and 3.2.1).

\section{Gene expression analysis of human renal biopsies}

Kidney biopsies were procured from patients with diabetic kidney disease from 2 cohorts (the European Renal cDNA Bank-Kroener-Fresenius biopsy bank (ERCB) and from Native Americans (Pima) enrolled in a randomized, placebo-controlled clinical trial to evaluate the renoprotective efficacy of losartan in type 2 diabetes (clinical trial registration number NCT00340678, clinicaltrials.gov) after informed consent and with approval of the local ethics committees. Patient characteristics are provided in Supplemental Table 2. Gene expression profiling of glomerular and tubulointerstitial compartments from microdissected biopsies was performed essentially as previously reported $(45,46)$. In brief, Affymetrix GeneChip Human Genome U133A 2.0 and U133 Plus 2.0 Array were used in this study. 
This analysis included only probesets that are present on both platforms. RMAexpress was used to perform robust multichip analysis, including background adjustment, quartile normalization, and summarization. The expression values are reported as log2-transformed intensity.

\section{Mouse studies}

C57BL/6 wild-type mice (Charles River) and BKS.Cg-m ${ }^{+/+}$Leprdb/BomTac (db/db) (Taconic, and Jackson Labs) were kept under normal housing conditions under a 12-hour light/dark cycle. Water and standard chow food were available ad libitum, except for a 4-hour fasting prior to capillary glucose measurement every 2 weeks. In selected studies, diabetic mice underwent uninephrectomy at 7 weeks of age, performed under general anesthesia, and started a high-fat diet at 10 weeks of age. Starting from week 16, mice received intraperitoneal injections on alternate days throughout 4 weeks with either 20 $\mathrm{mg} / \mathrm{kg}$ MDM2 inhibitor Nutlin-3a (Selleckchem) in 50\% DMSO (vehicle) or vehicle only. Mice were sacrificed after 4 weeks of treatment. Plasma and urine were collected before the treatment and in the course of the treatment, after 2 and 4 weeks. All experiments involving mice were conducted according to German and USA animal protection laws and approved by the local government authorities.

The podocyte-specific $M D M 2$-knockout mice were generated by crossing $M D M 2^{\mathrm{f} / \mathrm{fl}}$ mice, in which the loxP sites were inserted into the $M D M 2$ gene, with $\mathrm{Nphs}_{-} \mathrm{Cre}^{+}$mice that constitutively express Cre recombinase under the control of the podocin promoter, as previously described (27). The $M D M 2^{\mathrm{t} / \mathrm{fl}}$ littermates, lacking the podocyte-Cre transgene, were used as control mice. To obtain tubule-specific MDM2-knockout mice, the $M D M 2^{\mathrm{t} / \mathrm{fl}}$ mice were bred with Pax8-rtTA;TetO-Cre ${ }^{+}$mice. The Pax8-rtTA;TetO-Cre ${ }^{+}$mice express the reverse tetracycline-dependent transactivator (rtTA) under the control of the Pax8 promoter, which upon doxycycline administration binds the tetracycline-responsive element of the Cre gene and drives Cre induction in renal tubular cells. Thus, the MDM2 deletion in the renal tubular cells occurs in $M D M 2^{\mathrm{t} / \mathrm{fl}} ;$ Pax8-rtTA;TetO-Cre ${ }^{+}$mice only upon doxycycline treatment. $M D M 2^{\mathrm{t} / \mathrm{fl}}$ mice lacking the Pax8rtTA; TetO-Cre ${ }^{+}$elements were used as controls.

Murine embryonic kidneys were dissected under sterile conditions from embryonic day 17.5 pregnant B6 mice and the kidneys were grown on 0.45 - $\mu \mathrm{m}$ polyethylene terephthalate membranes in 6 -well multiwell plates in a $37^{\circ} \mathrm{C}$ incubator for 5 days as described previously (47). Explants were grown in Dulbecco's modified Eagle's medium-Ham's F-12 nutrient mixture (DMEM-F12) with Richter's modification (Life Technologies) containing $50 \mathrm{mg} / \mathrm{ml}$ transferrin (Sigma-Aldrich) and either DMSO or 5 $\mu \mathrm{M}$ Nutlin-3a (Selleckchem, catalog S8059) for 5 days. Media were not changed during each experiment. Explants were fixed in 4\% formaldehyde for 10 minutes, washed with PBS, and then embedded in paraffin. Tissue sections $(5 \mu \mathrm{m})$ were stained with periodic acid-Schiff (PAS) and imaged by light microscopy. Immunofluorescent staining of WT1 in the cryosections was carried out as described (48). Briefly, sections were incubated overnight at $4^{\circ} \mathrm{C}$ with antibodies against WT1, washed with PBS, and incubated for 1 hour at room temperature with Alexa Fluor-conjugated secondary antibodies and visualized by a BIOREVO BZ-9000 fluorescence microscope (Keyence).

The urine ACR was measured with the albumin ELISA kit (Bethyl Laboratories) and creatinine kit (DiaSys), according to the manufacturers' instructions. BUN was assessed with a colorimetric assay (DiaSys) and calculated as $\mathrm{mg} / \mathrm{dl}$. Urine metabolomics was performed as per our recent publications $(3,48)$.

\section{Renal pathology assessment}

Kidney tissue was fixed in $4 \%$ neutral buffered formalin, dehydrated in a graded alcohol series, and embedded in paraffin. The sections were deparaffinized, rehydrated, transferred into citrate buffer, and microwave treated for antigen retrieval and processed as described for immunohistochemistry (36). The following antibodies were used: guinea pig anti-mouse nephrin (1:100, catalog BP5030, Acris Antibodies), rabbit anti-MDM2 (1:100, ab38618, Abcam), rabbit anti-mouse TP53 (1:500, catalog VP-P956, Vector Laboratories), and rabbit anti-mouse WT1 (1:25; catalog sc-192, Santa Cruz Biotechnology). Immunofluorescent staining was evaluated using an LSM 510 confocal microscope and LSM software (Carl Zeiss, AG). Human kidney slides were obtained from one of the co-authors (VD) and processed for MDM2 immunostaining in a protocol similar to that described above for mouse tissues. 


\section{Statistics}

Functional enrichment analysis. BiNGO (15), one of the Cytoscape Apps, was used on Cytoscape 3.2.1 to identify enriched biological processes and cellular component based on GO in our MetBridge ${ }_{\mathrm{DKD}}$ network. Let $t \in T$ be set of GO terms for biological processes or cellular components. Let $p \in P$ be set of proteins in our whole MetBridge network (reference set), and $p \in P_{13}$ be those in the MetBridge $\mathrm{DKD}_{\mathrm{D}}$ network $\left(P_{13} \subset P\right)$. Let $p \in M(t)$ be a set of proteins that are associated with term $t$. Each $p$ may belong to some $M(t)$, i.e., $p \in M\left(t_{1}\right), p \in M\left(t_{2}\right), \cdots p \in M\left(t_{n}\right)$, where $0 \leq n \leq|T|$. BiNGO tested whether the rate of proteins having the given GO term $t$ in MetBridge ${ }_{\mathrm{DKD}}, r_{13}=\left(\left|p \in P_{13} \wedge p \in M(t)\right|\right) /\left|P_{13}\right|$, is larger than that in MetBridge, $r=(|p \in P \wedge p \in M(t)|) /|P|$, where $H_{0}: r_{13}=r, H_{1}: r_{13}>r$. The hypergeometric tests were done for all GO terms $t \in T$ that belong to biological processes or cellular component in the test set, followed by Benjamini and Hochberg multiple testing correction. Default settings of BiNGO were used where possible. Similar analysis was done for the enzymes.

\section{Gene expression patterns in human renal biopsies}

We investigated the expression levels of the top 5 genes in glomeruli and tubulointerstitium. These genes came out of our network analyses. For each gene, we calculated $P$ values based on the differences in the expression levels among living donors, ERCB, and Pima Native Americans cohorts (living donors versus ERCB and living donors versus Pima Native Americans) using $t$ tests. We further marked $P$ values that were still significant after adjusting for multiple comparisons (Bonferroni corrected $P$ value $<0.05$ ) due to 10 tests within each validation cohort.

\section{Mouse group comparison}

Comparison between 2 groups shown in Figures 4 and 5 was performed by 2-tailed $t$ test when groups were larger than $5(n>5)$ and by Mann-Whitney $U$ test (Wilcoxon rank sum test) when one of the groups or both were smaller, except for comparison of ACRs, where Welch's 1-tailed $t$ test was used for comparison owing to inherent variability of albuminuria measurements (Figure $4 \mathrm{C}$ ). A value of $P<0.05$ was considered to be statistically significant. All statistical analyses were performed using GraphPad Prism or R.

\section{Study approval}

All human studies have been approved by the IRB of the University of California, San Diego (UCSD), University of Michigan, Columbia University, and the NIDDK Intramural Research Program. All animal studies at UCSD and Nephrologisches Zentrum, Medizinische Klinik und Poliklinik IV, Klinikum der Universität were approved by the IUCAC and Committee on Research Animal Care and Regierungspräsidium Oberbayern, respectively.

\section{Author contributions}

RS did bioinformatics/network analyses, assisted with immunostaining, and prepared the manuscript. RS and $\mathrm{KO}$ developed Cytoscape App. ARA conducted adult mice experiments and supported setting the protocols for the embryonic mice experiments. YHY, JP, and SM conducted mice experiments. MD and BVE performed the metabolomics analysis and data processing. MP and LN supported statistical analyses. WJ and MK supported gene expression analyses from the human kidney biopsy datasets, which were obtained from RN. DT provided the measurements in the MDM2 KO mice. SR and SRM supported the adult mice experiments. VD provided kidney tissue sections from patients and interpreted the immunostaining results. TI provided bioinformatics tools and provided expertise for the Cytoscape analysis. JCIB and EB provided expertise on mouse embryonic kidney studies. HJA provided support and guidance for the project. KS supervised the overall project and edited the manuscript.

\section{Acknowledgments}

We thank Jane Kim for helpful discussions. We thank Susumu Goto for support and advice on KEGG data usage. We are also thankful to SK Devarapu for guidance and counseling. We thank Ms. Larkin Slater for her support on mice experiments.

RS, LN, MP, MD, BVE, YHY, SM, and KS are all supported in part by NIH grant DP3 DK094352 to KS. RS, KO, and TI are also funded by the National Resource for Network Biology (P41 GM103504) and the 
San Diego Center for Systems Biology (P50 GM085764). WJ and MK are supported by P30DK081943 (University of Michigan O'Brien Kidney Translational Core Center). KS was also supported by grants from the Juvenile Diabetes Research Foundation and a VA Merit Award 5101BX000277. The work of DT and HJA was supported by grants from the Deutsche Forschungsgemeinschaft TH 1836/1-2 and AN372/11-2. $\mathrm{RN}$ was supported by the Intramural Research Program of the National Institute of Diabetes and Digestive and Kidney Diseases.

Address correspondence to: Kumar Sharma, Professor of Medicine, Director, Center for Renal Translational Medicine, Director, Institute of Metabolomic Medicine, University of California, San Diego, 9500 Gilman Drive, La Jolla, California 92093-0711, USA. Phone: 858.822.0870; E-mail: kumarsharma@ucsd.edu.

1. Patti GJ, Yanes O, Siuzdak G. Innovation: Metabolomics: the apogee of the omics trilogy. Nat Rev Mol Cell Biol. 2012;13(4):263-269.

2. Hirayama A, et al. Metabolic profiling reveals new serum biomarkers for differentiating diabetic nephropathy. Anal Bioanal Chem. 2012;404(10):3101-3109.

3. Sharma K, et al. Metabolomics reveals signature of mitochondrial dysfunction in diabetic kidney disease. J Am Soc Nephrol. 2013;24(11):1901-1912.

4. Soga T, et al. Differential metabolomics reveals ophthalmic acid as an oxidative stress biomarker indicating hepatic glutathione consumption. J Biol Chem. 2006;281(24):16768-16776.

5. Kanehisa M, Goto S. KEGG: kyoto encyclopedia of genes and genomes. Nucleic Acids Res. 2000;28(1):27-30.

6. Joshi-Tope G, et al. Reactome: a knowledgebase of biological pathways. Nucleic Acids Res. 2005;33(Database issue):D428-D432.

7. Gao J, et al. Metscape: a Cytoscape plug-in for visualizing and interpreting metabolomic data in the context of human metabolic networks. Bioinformatics. 2010;26(7):971-973.

8. Kankainen M, Gopalacharyulu P, Holm L, Oresic M. MPEA--metabolite pathway enrichment analysis. Bioinformatics. 2011;27(13):1878-1879.

9. Xia J, Wishart DS. MSEA: a web-based tool to identify biologically meaningful patterns in quantitative metabolomic data. Nucleic Acids Res. 2010;38(Web Server issue):W71-W77.

10. Orth JD, Palsson BØ. Systematizing the generation of missing metabolic knowledge. Biotechnol Bioeng. 2010;107(3):403-412.

11. Krogan NJ, et al. Global landscape of protein complexes in the yeast Saccharomyces cerevisiae. Nature. 2006;440(7084):637-643.

12. Stark C, Breitkreutz BJ, Reguly T, Boucher L, Breitkreutz A, Tyers M. BioGRID: a general repository for interaction datasets. Nucleic Acids Res. 2006;34(Database issue):D535-D539.

13. Shannon P, et al. Cytoscape: a software environment for integrated models of biomolecular interaction networks. Genome Res. 2003;13(11):2498-2504.

14. Kann M, et al. Genome-wide analysis of Wilms' tumor 1-controlled gene expression in podocytes reveals key regulatory mechanisms. J Am Soc Nephrol. 2015;26(9):2097-2104.

15. Maere S, Heymans K, Kuiper M. BiNGO: a Cytoscape plugin to assess overrepresentation of gene ontology categories in biological networks. Bioinformatics. 2005;21(16):3448-3449.

16. Batch BC, Hyland K, Svetkey LP. Branch chain amino acids: biomarkers of health and disease. Curr Opin Clin Nutr Metab Care. 2014;17(1):86-89.

17. Li M, et al. GC/TOFMS analysis of metabolites in serum and urine reveals metabolic perturbation of TCA cycle in $\mathrm{db} / \mathrm{db}$ mice involved in diabetic nephropathy. Am J Physiol Renal Physiol. 2013;304(11):F1317-F1324.

18. Habib SL. Diabetes and renal tubular cell apoptosis. World J Diabetes. 2013;4(2):27-30.

19. Marshall CB, Shankland SJ. Cell cycle and glomerular disease: a minireview. Nephron Exp Nephrol. 2006;102(2):e39-e48.

20. Kuan CJ, al-Douahji M, Shankland SJ. The cyclin kinase inhibitor p21WAF1, CIP1 is increased in experimental diabetic nephropathy: potential role in glomerular hypertrophy. J Am Soc Nephrol. 1998;9(6):986-993.

21. Bandyopadhyay S, et al. Rewiring of genetic networks in response to DNA damage. Science. 2010;330(6009):1385-1389.

22. Jeong H, Mason SP, Barabási AL, Oltvai ZN. Lethality and centrality in protein networks. Nature. 2001;411(6833):41-42.

23. Lecker SH, Mitch WE. Proteolysis by the ubiquitin-proteasome system and kidney disease. J Am Soc Nephrol. 2011;22(5):821-824.

24. Rakhshandehroo M, Knoch B, Müller M, Kersten S. Peroxisome proliferator-activated receptor alpha target genes. PPAR Res. 2010.

25. Shadfan M, Lopez-Pajares V, Yuan ZM. MDM2 and MDMX: Alone and together in regulation of p53. Transl Cancer Res. 2012;1(2):88-89.

26. Nag S, Qin J, Srivenugopal KS, Wang M, Zhang R. The MDM2-p53 pathway revisited. J Biomed Res. 2013;27(4):254-271.

27. Thomasova D, et al. Murine double minute-2 prevents p53-overactivation-related cell death (podoptosis) of podocytes. $J A m$ Soc Nephrol. 2015;26(7):1513-1523.

28. Brown CJ, Lain S, Verma CS, Fersht AR, Lane DP. Awakening guardian angels: drugging the p53 pathway. Nat Rev Cancer. 2009;9(12):862-873.

29. Shen H, Maki CG. Pharmacologic activation of p53 by small-molecule MDM2 antagonists. Curr Pharm Des. 2011;17(6):560-568

30. Tovar C, et al. Small-molecule MDM2 antagonists reveal aberrant p53 signaling in cancer: implications for therapy. Proc Natl Acad Sci U S A. 2006;103(6):1888-1893.

31. Vassilev LT, et al. In vivo activation of the p53 pathway by small-molecule antagonists of MDM2. Science. 2004;303(5659):844-848.

32. Hilliard SA, Yao X, El-Dahr SS. Mdm2 is required for maintenance of the nephrogenic niche. Dev Biol. $2014 ; 387(1): 1-14$.

33. Montes de Oca Luna R, Wagner DS, Lozano G. Rescue of early embryonic lethality in mdm2-deficient mice by deletion of 
p53. Nature. 1995;378(6553):203-206.

34. Wang TJ, et al. Metabolite profiles and the risk of developing diabetes. Nat Med. 2011;17(4):448-453.

35. Oliver S. Guilt-by-association goes global. Nature. 2000;403(6770):601-603.

36. Mulay SR, Thomasova D, Ryu M, Anders HJ. MDM2 (murine double minute-2) links inflammation and tubular cell healing during acute kidney injury in mice. Kidney Int. 2012;81(12):1199-1211.

37. Baykal T, et al. Consanguineous 3-methylcrotonyl-CoA carboxylase deficiency: early-onset necrotizing encephalopathy with lethal outcome. J Inherit Metab Dis. 2005;28(2):229-233.

38. Niewczas MA, et al. Uremic solutes and risk of end-stage renal disease in type 2 diabetes: metabolomic study. Kidney Int. 2014;85(5):1214-1224

39. Donati G, Peddigari S, Mercer CA, Thomas G. 5 S ribosomal RNA is an essential component of a nascent ribosomal precursor complex that regulates the Hdm2-p53 checkpoint. Cell Rep. 2013;4(1):87-98.

40. Hershkovitz D, Burbea Z, Skorecki K, Brenner BM. Fetal programming of adult kidney disease: cellular and molecular mechanisms. Clin J Am Soc Nephrol. 2007;2(2):334-342.

41. Moritz KM, Dodic M, Wintour EM. Kidney development and the fetal programming of adult disease. Bioessays. $2003 ; 25(3): 212-220$.

42. Zandi-Nejad K, Luyckx VA, Brenner BM. Adult hypertension and kidney disease: the role of fetal programming. Hypertension. 2006;47(3):502-508

43. Baumgart E, et al. Mitochondrial alterations caused by defective peroxisomal biogenesis in a mouse model for Zellweger syndrome (PEX5 knockout mouse). Am J Pathol. 2001;159(4):1477-1494.

44. Hwang I, et al. Catalase deficiency accelerates diabetic renal injury through peroxisomal dysfunction. Diabetes. 2012;61(3):728-738

45. Ju W, et al. Renal gene and protein expression signatures for prediction of kidney disease progression. Am J Pathol. 2009;174(6):2073-2085

46. Hodgin JB, et al. Identification of cross-species shared transcriptional networks of diabetic nephropathy in human and mouse glomeruli. Diabetes. 2013;62(1):299-308.

47. Barak H, Boyle SC. Organ culture and immunostaining of mouse embryonic kidneys. Cold Spring Harb Protoc. 2011;2011(1):pdb.prot5558

48. You YH, Quach T, Saito R, Pham J, Sharma K. Metabolomics reveals a key role for fumarate in mediating the effects of NADPH oxidase 4 in diabetic kidney disease. J Am Soc Nephrol. 2016;27(2):466-481. 